Instituto Internacional de Investigación y Desarrollo Tecnológico Educativo INDTEC, C.A.

DOI: https://doi.org/10.29394/Scientific.issn.2542-2987.2019.4.14.10.205-227

OAI-PMH: http://www.indteca.com/ojs/index.php/Revista Scientific/oai

Artículo Original / Original Article

\title{
El conectivismo y las TIC: Un paradigma que impacta el proceso enseñanza aprendizaje
}

Autores: Jorge Luís Cueva Delgado Universidad Nacional de Educación, UNAE cueva8387@gmail.com Azogues, Ecuador https://orcid.org/0000-0001-8760-6854

Arelys García Chávez Universidad Nacional de Educación, UNAE arelys.garcia@unae.edu.ec

Azogues, Ecuador https://orcid.org/0000-0003-3730-0499

Oscar Antonio Martínez Molina Universidad Nacional de Educación, UNAE oscar.martinez@unae.edu.ec Azogues, Ecuador https://orcid.org/0000-0003-1123-5553

\section{Resumen}

El artículo es resultado de una tarea del Grupo de Investigación en educación y tecnología, desarrollado en la Universidad Nacional de Educación de Ecuador (UNAE), específicamente de la Línea de investigación Tecnología e innovación Educativa, del grupo de investigación en Educación y Tecnología (GIET); en el artículo se evidencian los fundamentos didácticos del uso de las TIC en el proceso de enseñanza aprendizaje; se asumen posturas teóricas que permiten asirse de los elementos esenciales del conectivismo como enfoque pedagógico para el uso de las TIC en el proceso de enseñanza aprendizaje. En este trabajo se analizan diferentes concepciones sobre el aprendizaje y se presenta una propuesta fundamentada en el conectivismo y la teoría de la actividad. Se propone un modelo de la influencia del conectivismo para el uso de las TIC en el proceso de enseñanza aprendizaje, en el que se establecen nuevas relaciones entre los elementos que componen el conectivismo y las categorías del proceso de enseñanza aprendizaje desarrollador, en el marco del buen vivir, así como, que se describen los nuevos rasgos que caracterizan al modelo y se define como cualidad resultante entre las relaciones de los componentes del modelo pedagógico de Aprendizaje Emprendedor.

Palabras clave: tic; enseñanza; aprendizaje.

Cómo citar este artículo:

Cueva, J., García, A., \& Martínez, O. (2019). El conectivismo y las TIC: Un paradigma que impacta el proceso enseñanza aprendizaje. Revista Scientific, 4(14), 205-227, e-ISSN: 2542-2987. Recuperado de: https://doi.org/10.29394/Scientific.issn.2542-2987.2019.4.14.10.205-227

Fecha de Recepción: 13-07-2019
Fecha de Aceptación: 30-09-2019
Fecha de Publicación: 05-11-2019 


\title{
Connectivism and ICT: A paradigm that impacts the teaching-learning process
}

\begin{abstract}
The article is the result of a task of the Research Group in education and technology, developed at the National University of Education of Ecuador (UNAE), specifically of the Research Line Technology and Educational Innovation, of the research group in Education and Technology (GIET); The article demonstrates the didactic foundations of the use of ICT in the teachinglearning process; Theoretical positions are assumed that allow us to grasp the essential elements of connectivism as a pedagogical approach to the use of ICT in the teaching-learning process. In this work, different conceptions about learning are analyzed and a proposal based on connectivism and activity theory is presented. A model of the influence of connectivism for the use of ICT in the teaching-learning process is proposed, in which new relationships are established between the elements that make up the connectivism and the categories of the developer-learning teaching process, within the framework of good living, as well as, that the new features that characterize the model are described and defined as the resulting quality between the relationships of the components of the pedagogical model of Entrepreneurial Learning.
\end{abstract}

Keywords: ict; teaching; learning.

\section{How to cite this article:}

Cueva, J., García, A., \& Martínez, O. (2019). Connectivism and ICT: A paradigm that impacts the teaching-learning process. Revista Scientific, 4(14), 205-227, e-ISSN: 2542-2987. Recovered from: https://doi.org/10.29394/Scientific.issn.2542-2987.2019.4.14.10.205-227

Date Received:

13-07-2019
Date Acceptance:

30-09-2019
Date Publication:

05-11-2019 


\section{Introducción}

La concepción de las sociedades desarrolladas con alta demanda en el uso de las Tecnologías de la Información y la Comunicación (TIC) que hoy se divisa, es producto del acelerado desarrollo y explosión de la evolución de la ciencia; la que está sustentada en las ansias del saber, conocer, dominar y buscar mejores resultados en los procesos productivos y sociales del hombre a lo largo de la historia.

El desarrollo de las TIC impulsado por la era digital produce cambios crecientes en las sociedades, varió la forma de las personas relacionarse, de comunicarse, de realizar las gestiones, de comportarse y hasta de divertirse, estos cambios imperantes en el contexto de la sociedad actual, demandan de la educación nuevos retos para enfrentar este fenómeno tecnológico, debido a que los niños de hoy desde que nacen están bajo la influencia directa de las enseñanzas de los medios tecnológicos, influencia que en el mejor de los casos es adecuada, pero que en otros, es nociva para el desarrollo psicosocial.

Se evidencian casos de niños y adolescentes que no se relacionan presencialmente con sus familiares y compañeros, nos les interesa el desarrollo de una vida en la que se intercambien informaciones, ideas, conocimientos, valores, afectos o simplemente los juegos de forma presencial, sino que están influenciado por las informaciones que son trasmitidas por el uso de los diferentes medios tecnológicos.

Los investigadores de este artículo consideran que la educación desempeña una dinámica importante en la preparación de estudiantes para un adecuado uso de las TIC en el proceso de enseñanza aprendizaje, y por ende, lograr mayores resultados cognitivos y un adecuado desarrollo de la personalidad, con el fin de prepararlos desde la vida, para la vida y en la vida. Por tal motivo es que los docentes deben tener una adecuada preparación en el uso de las TIC para el desarrollo de un proceso de enseñanza aprendizaje de calidad. 
En este sentido, autores como Castellanos, Castellanos, Llivina, Silverio, Reinoso y García (2002); Zilberstein (2012); Rico y Silvestre (2003); Rico, Santos y Martín-Viaña (2004); Silvestre (2012); Santos (2013): plantean rasgos esenciales para lograr un aprendizaje desarrollador en los estudiantes, que les permita el constante autodesarrollo, regulación e independencia cognoscitiva como muestra de un verdadero proceso de enseñanza aprendizaje en el que se empleen diferentes medios tecnológicos.

Por otro lado, en el Manual de Metodología Didáctica de la Unidad de promoción y Desarrollo (2013), se plantea que:

El aprendizaje es un proceso y tiene lugar cuando la persona en virtud de determinadas experiencias que incluyen necesariamente interacciones con el entorno, produce: respuestas nuevas, o modifica las ya existentes, o cuando algunas actividades ya existentes se emiten con relación a aspectos nuevos del contexto, es decir, cuando el individuo establece nuevas relaciones entre su actividad y el entorno (pág. 6).

Otra definición importante de aprendizaje, es la aportada por Keller (2013), al plantear que: "el aprendizaje es visto como un proceso que ocurre al interior de ambientes difusos de elementos centrales cambiantes, que no necesariamente están bajo control del individuo" (párr. 5).

Al analizar la definición antes mencionadas se infiere que el aprendizaje es influenciado por el entorno y los ambientes de aprendizaje que influyen sobre el sujeto, no siempre estos están controlados por el aprendiz, las dificultades que se le presentan crean situaciones que obligan a buscar soluciones que le permitan la adquisición de un nuevo conocimiento.

Para los autores de este artículo el proceso de enseñanza aprendizaje se desarrolla gradualmente cuando las necesidades en base a las experiencias de los sujetos y mediante la interacción constante y directa con el medio que lo rodea, y la influencia del contexto le exige buscar respuestas 
y soluciones a las problemáticas que se les presentan en la dinámica del desarrollo, para lograr la transformación del entorno y de su situación, ya sea personal o social.

El objetivo es proponer un modelo de la influencia del conectivismo para el uso de las TIC en el proceso de enseñanza aprendizaje, en el que se establecen nuevas relaciones entre los elementos que componen el conectivismo y las categorías del proceso de enseñanza aprendizaje desarrollador, en el marco del buen vivir.

\section{Desarrollo}

El proceso de enseñanza aprendizaje es vital para lograr el desarrollo de la personalidad de los estudiantes, para la formación de la sociedad a la que se aspira, es por ello, que es una categoría polisémica y de mucho interés para los pedagogos, tal es el caso de Reinoso, Ballester, González, Torres y Ribot (2013), al decir que:

El aprender a conocer implica apropiarse de procedimientos y estrategias cognitivas, metacognitivas y motivacionales que permitan producir el saber, resolver problemas, aprender a aprender de manera permanente, a lo largo de toda la vida en diferentes contextos y situaciones. Presuponen que los niños, adolescentes y jóvenes se apropien de los instrumentos intelectuales necesarios para pensar, reflexionar, buscar información, valorarla y utilizarla en un mundo en constante cambio. Todo esto forma parte de procesos que están en la base de aprendizajes desarrolladores (pág. 33).

Para este autor en el proceso de adquisición del conocimiento el estudiante debe apropiarse de las herramientas intelectuales y de los medios que tiene a su disposición para resolver las dificultades que se les presentan en el desarrollo de su vida, por lo que los docentes deben centrar el proceso de enseñanza-aprendizaje en buscar cómo formar estudiantes con las habilidades necesarias para que mediante el empleo de las TIC y otros medios 
gestionen sus propios conocimientos, que les permitan la solución de sus necesidades tanto cognitivas como afectiva motivacionales.

Para los autores de este artículo el proceso de enseñanza aprendizaje activo y desarrollador es aquel que ofrece la posibilidad a los estudiantes de ser creativos, dinámicos, que gestionen sus propios conocimientos, que se motiven y le impregnen significado al proceso mediante el cual los sujetos se aprehenden de los conocimientos, sentimientos y valores que la sociedad en contextos históricos concretos les ha legado, este proceso es influenciado por leyes y por la constante interacción y retroalimentación social que permite el crecimiento personal, espiritual y social.

En tal sentido se infiere que la tarea de aprendizaje debe estar estructurada con un fin y propósito para que se logren los objetivos planteados y brindarles a los estudiantes las herramientas necesarias para desarrollarse y enfrentarse a las diferentes problemáticas en el ámbito educativo y social.

El proceso de aprendizaje ocurre a lo largo de la vida, desde que el niño nace comienza a aprender, unas veces por instinto, otras por imitación, cuando juegan aprenden rápidamente imitando a las personas mayores, desarrolla el juego simbólico y empieza a desarrollar la creatividad, imaginación y la formación de la personalidad entre otros procesos importantes.

Al interactuar y palpar los objetos recibe una información que le permite realizar representaciones mentales y conocer el medio en el que se desarrolla, es por ello que varios autores le atribuyen gran importancia a los medios de enseñanza y aprendizaje para el desarrollo de los niños y niñas,

\subsection{El conectivismo una teoría para enriquecer el proceso de enseñanza} aprendizaje.

Desde que las sociedades evolucionaron centraron la atención en la educación con intereses propios influenciados por los contactos y modos de vida de cada sociedad en una época precisa, con el objetivo de formar 
personas que las sociedad necesitaba, evolucionan los diferentes enfoques educativos, pero en las últimas décadas con el desarrollo constante y a ritmo creciente, se crean y desarrollan las Tecnologías de la Información y la Comunicación, dando paso a una nueva tendencia o sociedad del conocimiento, sociedad de la información o era digital como algunos investigadores refieren.

Sin ánimo de entrar en elementos esenciales de conceptualización en este artículo se asevera desde las experiencias e investigaciones de los autores que la sociedad del conocimiento entendida según Pedraja (2017), citado por Pérez, Mercado, Martínez, Mena y Partida (2018), impone:

Exigencias crecientes para las naciones, las organizaciones y las personas. En efecto, cada vez se torna más elevada la preparación intelectual necesaria para desempeñarse con eficacia social en una sociedad digital y de redes que interactúan de manera sistemática e ininterrumpida (pág. 6).

Según la definición antes citada las organizaciones y las personas deben tener una eficiente preparación para desempeñarse adecuadamente en la sociedad, las TIC son herramientas que facilitan el acceso al conocimiento ubicuo y optimizan los procesos de adquisición de la información, estas tecnología modificaron las relaciones humanas, sus ambientes amenos y acogedores invitan a su uso, despiertan el interés y la motivación, las conexiones que se establecen en las diferentes redes y nodos acortan las barreras de tiempo y espacio.

A consideración de los autores de este artículo el conectivismo es un enfoque pedagógico que sí se aplica consecuentemente y de forma organizada, permite el empleo de las tecnologías en el proceso de enseñanza aprendizaje, para obtener excelentes resultados, sin perder el horizonte de que el rol protagónico es de los estudiantes, las TIC solo son los medios o recursos que facilitarán y mediarán el acceso al conocimiento. 
El conectivismo, para Siemens (2004a): es "la integración de principios explorados por las teorías del caos, redes, complejidad y auto-organización" (pág. 6); Según esta definición el conectivismo asume las complejidades que se desarrollan constantemente en todos los procesos, es decir, la educación es un proceso complejo en el que se presentan constantes barreras a superar, además, la teoría del caos considera que las objetos y fenómenos están interconectados entre sí, elementos que evidencian el carácter sistémico de las categorías de la didáctica, las que se deben desarrollar mediante el uso de las redes del conocimiento que propician los medios tecnológicos, elementos que permiten el desarrollo de la organización y autoorganización tanto de las instituciones como de los agentes que se desarrollan en ellas.

El conectivismo se basa en los siguientes principios desarrollados por Siemens (2004b):

El aprendizaje y el conocimiento dependen de la diversidad de opiniones; El aprendizaje es un proceso de conectar nodos o fuentes de información especializados; El aprendizaje puede residir en dispositivos no humanos; La capacidad de saber más es más crítica que aquello que se sabe en un momento dado; La alimentación y mantenimiento de las conexiones es necesaria para facilitar el aprendizaje continuo; La habilidad de ver conexiones entre áreas, ideas y conceptos es una habilidad clave; La actualización (conocimiento preciso y actual) es la intención de todas las actividades conectivistas de aprendizaje; La toma de decisiones es, en sí misma, un proceso de aprendizaje (pág. 7).

Para aplicar un adecuado uso de las TIC en el proceso de enseñanza aprendizaje y obtener los resultados esperados es necesario tener en cuenta la correcta aplicación de los principios antes mencionados, porque los resultados dependerán de su correcta aplicación.

Es importante que tanto el docente como los estudiantes comprendan que para tener un adecuado aprendizaje se necesita interpretar la riqueza que contiene la diversidad de criterios, que es necesario facilitarles a los 
estudiantes las herramientas para que puedan interconectar las diferentes fuentes de conocimientos que propicien su desarrollo.

Figura 1. Modelo de la influencia del conectivismo para el uso de las TIC en el proceso de enseñanza aprendizaje.

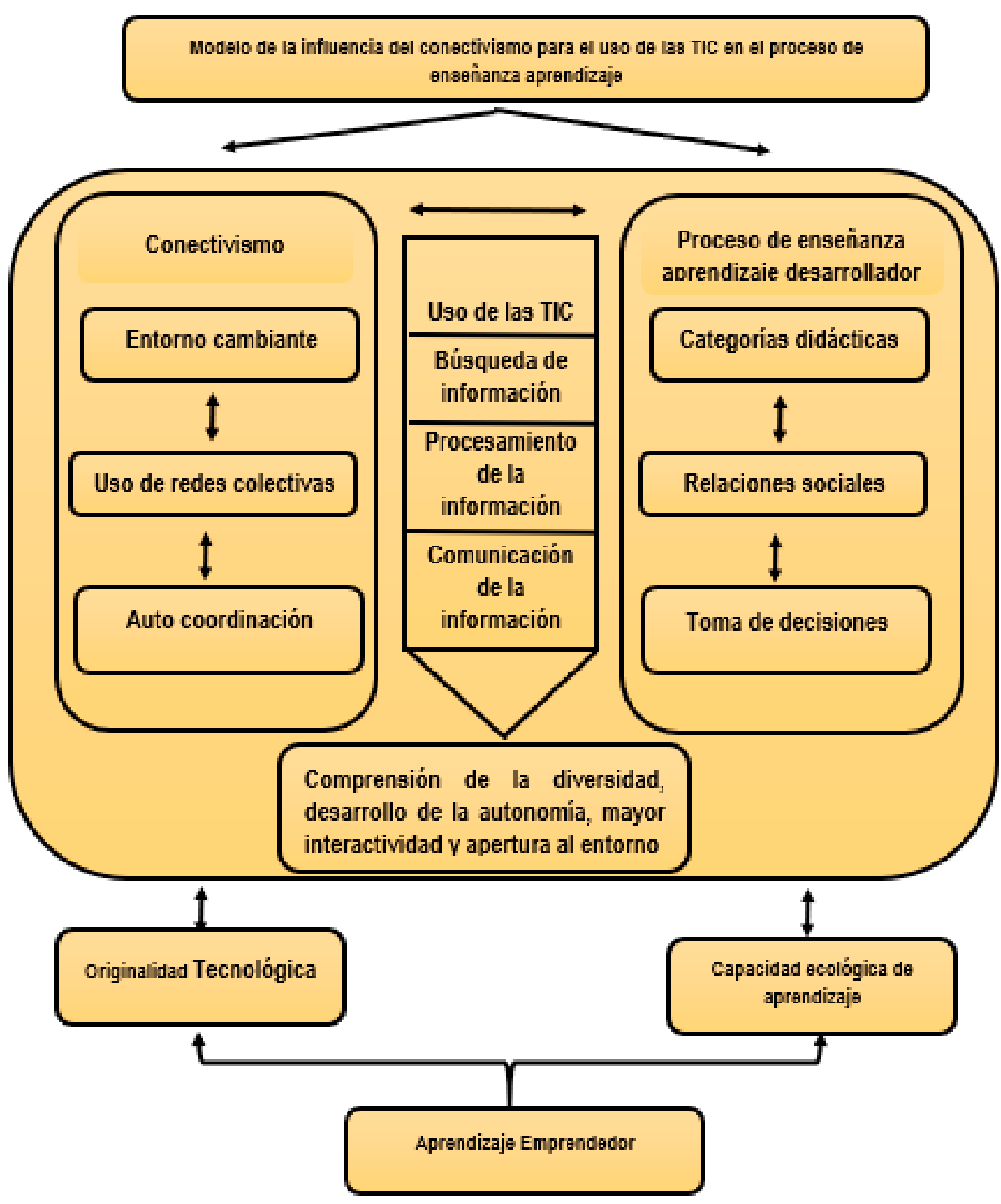

Fuente: Los Autores (2019).

A continuación, en la figura 1 , se procede a explicar la esencia de los componentes del modelo y las relaciones que se establecen entre ellos; para 
eso es necesario comprender qué es un modelo; asumiendo la definición aportada por Valledor et al. (2009), citado por Cueva (2016), al exponer que:

Un modelo es una representación (gráfica, teórica, matemática o material) de un objeto real mediante un análogo $\mathrm{y}$, al mismo tiempo, es el conjunto de ideas que se va conformando el investigador del objeto, ideas que se integran, incrementan, perfeccionan y enriquecen. Pero dicha representación se engendra en un proceso de abstracción-concreción, que parte de la información que dispone el investigador para concebir y modelar bien una concepción empírica o teórica del objeto (pág. 14).

Mediante la aplicación del modelo antes graficado se pretende elevar la preparación de los estudiantes para el uso de las TIC en el proceso de enseñanza aprendizaje, y así obtener mejores resultados académicos, además, se propicia el desarrollo de destrezas, habilidades, acciones, valores y emociones que le permiten desarrollarse con éxito en la vida. Para ello es necesario que al implementar el modelo se tenga en cuenta el componente del conectivismo (entorno cambiante, uso de redes colectivas y la autodeterminación).

A través de la incidencia del entorno cambiante se modifican las formas de solucionar los problemas y aparecen otros, a los que nunca nos habíamos enfrentados, estas entropías que se presentan en las relaciones sociales y en los procesos, además, se concretan en el proceso de enseñanza aprendizaje dando origen a nuevas dificultades y barreras, elementos que condicionan la constante preparación de los docentes y estudiantes para emplear nuevos métodos en aras de brindar soluciones a las necesidades de aprendizajes y en la búsqueda activa del conocimiento.

El contexto cambiante influye proporcionalmente en la calidad del aprendizaje, pues obliga a los sujetos del proceso de enseñanza aprendizaje a desarrollarse, a evolucionar, a buscar nuevas herramientas que le permitan el desarrollo de sus capacidades cognitivas, elemento que favorece la 
formación de una cultura investigativa con habilidades que le permite la búsqueda activa de la información para solucionar sus necesidades, sustentado en los diferentes estilos de aprendizaje.

El uso de las redes colectivas propicia el desarrollo de estrategias de aprendizaje, el empleo de recursos tecnológicos para la búsqueda activa del conocimiento que le permita solucionar las entropías a las que se enfrentan los estudiantes en el actuar cotidiano, con el desarrollo de las TIC y su empleo en el proceso de enseñanza aprendizaje se crean redes colectivas de conocimiento, se desarrollaron las fuentes de la información mediante las que los estudiantes se nutren del conocimiento que necesitan.

Las redes colectivas les brindan la posibilidad a los estudiantes de crear y comunicar su conocimiento, así como, interactuar de forma virtual o en línea con la comunidad tecnológica que se encuentra interactuando con la información, así como, establecer ambientes de aprendizajes; elementos que le permiten a los estudiantes trazar su propia trayectoria de aprendizaje según sus necesidades.

Estas redes colectivas permiten acceder de forma inmediata a la información, así como, comprender y valorar que el aprendizaje colectivo es más rico que el individual, además, les posibilitan interiorizar y experimentar que el aprendizaje individual enriquece al aprendizaje colectivo.

Mediante el intercambio en estas redes se crea una cultura tecnológica y una identidad digital que los caracteriza como usuarios digitales, se apropian de las habilidades y estilos de buscar y crear conocimientos, desarrollan la ética digital que les permite reconocer el aporte colectivo a su crecimiento personal, aprender a respetar los criterios y la sapiencia de los demás, así como, enriquecer y argumentar sus puntos de vistas, posiciones y creencias, elementos que evidencian un crecimiento cognitivo y afectivo motivacional.

La auto coordinación es el proceso mediante el cual los estudiantes como producto de su metacognición planifican y aplican acciones que les 
permiten la búsqueda del conocimiento en los diferentes redes y fuentes para la satisfacción de sus necesidades educativas, o para la solución de las dificultades a las que se enfrentan en el actuar cotidiano tanto educativo como de relaciones sociales.

Este componente permite a los estudiantes desarrollar habilidades y destrezas para realizar acciones para valorar si la información que obtienen es útil o no, así como, el análisis de sus modos de actuación con la información y el conocimiento que adquieren y construyen.

Mediante la auto coordinación los estudiantes planifican su trayectoria de aprendizaje, así como, las acciones que le permiten un análisis y procesamiento de alto vuelo teórico para planificar y evaluar las acciones que realizan en aras de la búsqueda activa del conocimiento para su autonomía y enriquecimiento espiritual.

El proceso de auto coordinación le posibilita a los estudiantes seleccionar la secuencia de acciones a seguir para interactuar en las diferentes redes colectivas de aprendizaje, en aras de solucionar sus inquietudes, así como, la lógica a emplear para construir y comunicar el conocimiento, elementos que le permiten un desarrollo digital para enriquecer su cultura en escenarios y contextos digitales, como evidencia del crecimiento profesional que responde a las exigencias de la sociedad contemporánea del conocimiento.

El componente conectivismo, antes descrito, establece relaciones de coordinación, subordinación y complementariedad recíproca con el proceso de enseñanza aprendizaje desarrollador para el logro de las aspiraciones de formar escolares preparados integralmente para la transformación positiva de la sociedad en la que se desarrollan.

Las categorías de la didáctica se enriquecen y complementan con los elementos esenciales del conectivismo, al lograrse a mayor escala los objetivos propuestos tanto a nivel curricular como general, debido a que los 
estudiantes interactúan en las redes colectivas de conocimiento nutriéndose y aportando nuevas ideas en las diferentes áreas del conocimiento en la que gestionan sus aprendizajes, elemento que propician el cumplimiento de las aspiraciones sociales con mayor calidad, calidez y solidez.

El empleo de métodos creativos y activos basados en las tecnología posibilita la búsqueda activa del conocimiento, brindándole la posibilidad a los estudiantes de gestionar sus conocimientos de forma amena y emocionante, que invita al estudio, el descubrimiento de nuevas técnicas y estrategias de aprendizaje les permite el desarrollo de la metacognición, la independencia en la búsqueda del conocimiento, posibilita el desarrollo de habilidades para establecer la auto coordinación en espacios de aprendizajes virtuales y tecnológicos.

El entorno cambiante les posibilita el empleo de nuevos métodos de enseñanza aprendizaje para solucionar las entropías que se les presentan a los estudiantes en el proceso del desarrollo cognitivo, así como, la organización creativa e innovadora de espacios y ambientes que permiten la interacción dinámica con el contenido a aprender, les posibilita mayor fijación del conocimiento, espacios de aprendizajes que le añaden una valor agregado al proceso de enseñanza aprendizaje, al brindar la posibilidad de la enseñanza en ambientes virtuales y semipresenciales; el empleo de herramientas tecnológicas que acortan las barreras del tiempo y el espacio, así como, la interacción directa y personalizada con el contenido sustentado en los estilos de aprendizajes de los estudiantes.

Al interactuar los elementos del conectivismo con las categorías del proceso de enseñanza aprendizaje desarrollador que se integran en un sistema propicia que al enriquecerse uno muestra ventajas y resultados positivos en los otros, los medios de enseñanza y aprendizaje se fortalecen a medidas que se les atribuye esa función a herramientas y productos tecnológicos que no fueron diseñados para ello, al emplearlos para la 
búsqueda activa del conocimiento y la apropiación de los contenidos se desarrollan creativamente, enriquecen el proceso de aprendizaje, se innova en el uso y se desarrolla la creatividad.

Los estudiantes y profesores al emplear las tecnologías desarrollan habilidades y se convierten en creadores de medios de enseñanza aprendizaje, retroalimentando las funciones de cada agente del proceso de enseñanza aprendizaje.

Estos elementos posibilitan emplear creativamente la evaluación, ya sea en su dimensión diagnóstica, formativa o sumatoria, posibilita la comprensión y seguimiento no solo del desarrollo cognitivo de los estudiantes, además, brinda seguimiento a la formación de valores, sentimientos, creatividad, originalidad, flexibilidad de los estudiantes en sus modos de actuación, para elaborar y comunicar sus resultados y conocimientos.

Permite constatar el proceso de crecimiento personal de los estudiantes, así como, emplear nuevos instrumentos de evaluación, como son la observación del desempeño de los estudiantes en línea, mediante la realización de una actividad, o el análisis mediante la aplicación de test virtuales a los estudiantes para constatar cómo reaccionan ante un estímulo o situación determinada.

Además, propicia que los estudiantes presenten el conocimiento de diferentes formas creativas e innovadoras, se brinda la posibilidad a los estudiantes para que se autoevalúen; creando una verdadera participación activa en este proceso.

La interacción directa y consciente de los estudiantes con los elementos e influencia del conectivismo en el proceso de enseñanza aprendizaje, propicia el desarrollo de habilidades, actitudes, emociones y valores para el desarrollo social, les permite establecer mejores relaciones sociales tanto en los pequeños grupos como en el salón de clases, además, desenvolverse en los diferentes contextos de la vida. 
Les posibilita el desarrollo de nuevas relaciones sociales en grupos interconectados tecnológicamente, la creación de amistades, permite el intercambio de ideas, costumbres, valores, culturas, experiencias y emociones que contribuyen a la formación de la personalidad de los estudiantes.

Se crean nuevas formas y métodos de establecer relaciones sociales que enriquecen el proceso de enseñanza aprendizaje, se eleva el nivel de individualización de los estudiantes, así como, la trasmisión del aprendizaje individual y colectivo posibilita la objetivación de los conocimientos y socialización de experiencias y vivencias.

En estas nuevas y creativas formas de relaciones sociales propicia el intercambio de cultura, códigos lingüísticos, conocimientos, sentimientos y valores que posibilitan el desarrollo tanto cognitivo como afectivo motivacional de los agentes del proceso de enseñanza aprendizaje.

Las relaciones sociales que se establecen mediante las redes colectivas y el empleo de las tecnologías enriquecen las funciones de las agencias educativas, así como, que los agentes del aprendizaje pasan de ser objetos de aprendizaje a sujetos activos, desarrollando la creatividad y la imaginación, al emplear formas novedosas e innovadoras para establecer relaciones sociales y el intercambio de experiencias, información, conocimiento y valores; elementos que le atribuyen un nuevo valor agregado a las instituciones educativas y enriquecen el proceso de enseñanza aprendizaje al desarrollarse una pedagogía activa centrada en el estudiantes y los nuevos contextos de aprendizaje para preparar a la sociedad del futuro.

Estas interacciones entre los componentes del modelo establecen nuevas relaciones que le permiten a los estudiantes la toma de decisiones consciente, comprenden el contexto en el que se desarrollan, adquieran las habilidades y capacidades para analizar y comprender las causas y efectos que originan en las sociedades y las diferentes organizaciones, en las que interactúan los contextos cambiantes y las complejidades a las que se 
enfrentan.

Estos elementos mediante el empleo de las redes, brindan la posibilidad de interactuar con los medios tecnológicos humanizando y optimizando el proceso de búsqueda de información que les propicia el análisis consciente y crítico de las situaciones para tomar las decisiones correctas en el momento oportuno.

Las nuevas relaciones que se establecen en el modelo contribuyen a formar en los estudiantes la capacidad de análisis, propiciando el desarrollo de la metacognición, al facilitar que los estudiantes comprendan sus potencialidades y vulnerabilidades para enfrentarse y resolver las diferentes entropías a las que se enfrentan en la dinámica de la sociedad. Estas esencias los pone en capacidad de realizar análisis profundos para tomar las decisiones que le permitan el planeamiento de acciones que posibiliten resolver sus necesidades, tanto académicas como personales, brindándole la posibilidad de mantener una participación activa y consciente en el proceso de enseñanza aprendizaje.

La toma de decisiones conscientes es una habilidad que desarrollan los estudiantes mediante la interacción con los componentes del modelo, elementos que evidencian un verdadero aprendizaje desarrollador, al preparar a los estudiantes con las herramientas necesarias para establecer acciones como entes activos en el proceso educativo, superándose a sí mismos en cada momento, estableciendo metas y proyectos de vida, desarrollando la creatividad y la capacidad de innovar al buscar nuevas soluciones a dificultades que exige de ellos el desarrollo cognitivo para transformar la realidad en beneficio propio y de la sociedad.

Según lo planteado el proceso antes mencionado posibilita la conexión entre los estudiantes y las redes en las que están interconectados, se transmiten nuevos códigos, informaciones, experiencia, vivencias significativas, surgen formas de transmitir y consolidar conocimientos; 
elementos que le propician a los estudiantes una conexión directa con el medio social, expresar sus individualidades y la forma de interpretar los procesos y fenómenos para transformar la sociedad en la que se desarrollan, como muestra de un crecimiento tanto cognitivo como afectivo profesional.

Los procesos antes descritos contribuyen a que los estudiantes comprendan la diversidad, en un mundo cambiante, así como, que aprenden a valorar la riqueza cultural que propicia la diversidad. Además, posibilita el desarrollo de la autonomía mediante una mayor interactividad con el empleo de las TIC, aspecto que le permiten desarrollar habilidades para la apertura al entorno que los rodea, elementos que posibilitan la expresividad, la interconectividad, la exploración y enriquecimiento del entorno, la solidez de los argumentos y el desarrollo cognitivo para la toma de decisiones sustentadas en sus principios, valores y la influencia del entorno.

Las interacciones realizadas entre el componente del conectivismo (entorno cambiante, uso de redes colectivas y la auto coordinación); el componente del proceso de enseñanza aprendizaje desarrollador (categorías didácticas, relaciones sociales y toma de decisiones) y los procesos que se encuentran en la base el uso de las TIC (búsqueda de información, procesamiento de la información y la comunicación de la información) se establecen relaciones de retroalimentación y sinergia que facilitan el desarrollo de rasgos esenciales que personalizan el proceso modelado, los mismos son:

Originalidad tecnológica: capacidad que desarrollan los estudiantes para interpretar, procesar y comunicar la percepción de los diferentes contextos en los que interactúan ante una situación dada, individualidad con la que aplican los conocimientos adquiridos mediante las TIC y desde las TIC.

Capacidad ecológica de aprendizaje: habilidades que adquieren los estudiantes para identificar su trayectoria de aprendizaje, así como, para analizar y reconocer qué información necesitan, en qué fuente buscarla, cómo acceder a ella, cuándo gestionarla y para qué; además, de emplear los medios 
tecnológicos mediante los que se sientan identificados para aplicar los conocimientos aprendidos.

Los rasgos antes mencionados establecen relaciones dialécticas en su constante interacción permitiendo que la originalidad tecnológica les posibilite a los estudiantes aplicar significativamente según sus experiencias, vivencias e interpretación del conocimiento mediante el uso de los medios tecnológicos, para resolver sus principales necesidades, desarrollando un estilo propio de aprendizaje mediante la capacidad ecológica de aprendizaje.

Luego del análisis del proceso modelados se llega a la conclusión de que el aumento del consumo de las TIC en todas las esferas de la vida social impone la necesidad de transformar los sistemas educativos en aras de elevar la calidad de la educación, donde los estudiantes sean los principales protagonistas, esta aspiración se puede lograr con la aplicación consciente del proceso modelado.

\section{Conclusiones}

Con el presente artículo no se pretende brindar recetas acabadas sobre el uso de la TIC en el proceso de enseñanza aprendizaje, solo se propone un modelo de la influencia del conectivismo en la educación, se establecen las nuevas relaciones ente los componentes del modelo y los rasgos fundamentales que lo caracterizan.

La teoría propuesta posibilita implementar un proceso de enseñanza aprendizaje desarrollador y activo, en el que los estudiantes son los protagonistas por excelencia, elevando sus niveles de motivación, interés por la investigación, así como, las destrezas para interactuar con las tecnologías en busca de la información útil para solucionar sus inquietudes, así como, la comunicación de la misma mediante las redes colectivas, elementos que le permiten la interacción activa y la toma de decisiones acertadas. 


\section{Referencias}

Castellanos, D., Castellanos, B., Llivina, M., Silverio, M., Reinoso, C., \& García, C. (2002). Aprender y enseñar en la escuela. La Habana, Cuba: Editorial Pueblo y Educación.

Cueva, J. (2016). Las Tecnologías de la Información y la Comunicación para la Gestión del conocimiento en la Educación Primaria. Tesis presentada en opción al grado científico de Doctor en Ciencias Pedagógicas. Cuba: Universidad de las Tunas.

Keller, J. (2013). Conectivismo: Una teoría de aprendizaje para la era digital. Estados Unidos: Infogram. Recuperado de:

https://infogram.com/conectivismo-una-teoria-de-aprendizaje-para-laera-digital-1g6qo2qvwqvyp78

Pérez, R., Mercado, P., Martínez, M., Mena, E., \& Partida, J. (2018). La sociedad del conocimiento y la sociedad de la información como la piedra angular en la innovación tecnológica educativa. RIDE. Revista Iberoamericana para la Investigación y el Desarrollo Educativo, 8(16), 1-24, e-ISSN: 2007-7467. Recuperado de:

http://dx.doi.org/10.23913/ride.v8i16.371

Reinoso, C., Ballester, S., González, C., Torres, Y., \& Ribot, G. (2013). El aprendizaje desarrollador. Teoría y práctica en la formación de educadores. Curso 31, ISBN: 978-959-18-0865-3. La Habana, Cuba: Educación Cubana.

Rico, P., Santos, E., \& Martín-Viaña, V. (2004). Proceso de enseñanza aprendizaje desarrollador en la escuela primaria. Teoría y Práctica. La Habana, Cuba: Editorial Pueblo y Educación.

Rico, P., \& Silvestre, M. (2003). Proceso de enseñanza-aprendizaje. La Habana, Cuba: Editorial Pueblo y Educación.

Santos, E. (2013). Categorías del proceso de enseñanza aprendizaje. En: Proceso de enseñanza aprendizaje desarrollador en la escuela 
primaria. Teoría y Práctica. La Habana, Cuba: Editorial Pueblo y Educación.

Siemens, G. (2004a,b). Conectivismo: Una teoría de aprendizaje para la era digital. Chile: Ministerio del Interior y Seguridad Pública SENDA Consorcio de Universidades del Estado de Chile. Recuperado de: https://www.comenius.cl/recursos/virtual/minsal v2/Modulo 1/Recurso s/Lectura/conectivismo Siemens.pdf

Silvestre, M. (2012). Exigencias didácticas para dirigir un proceso de enseñanza-aprendizaje desarrollador y educativo. 1ra edición. [CDROM]. La Habana, Cuba: Ministerio de Educación de la República de Cuba.

Unidad de promoción y Desarrollo (2013). Manual de Metodología Didáctica. Almería, España: Junta de Andalucía - Diputación de Almería. Recuperado de:

https://issuu.com/verocedillo/docs/curso de metodolog a did ctica Zilberstein, J. (2012). Tendencias en la Didáctica. Sus categorías en una concepción desarrolladora de la escuela primaria. 1ra edición. [CDROM]. La Habana, Cuba: Ministerio de Educación de la República de Cuba. 
Nacido en Las Tunas, Cuba, el 7 de marzo del año

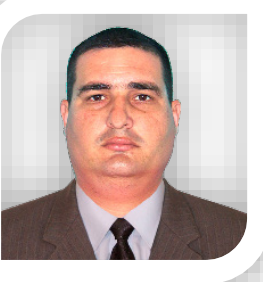
1983. Licenciado en Educación. Especialidad Informática; ostenta el Grado Científico de Doctor en Ciencias Pedagógicas; Comencé la vida profesional en el año 2000, en la Escuela Primaria Argentina, Cuba; Director de la Escuela Primaria Osvaldo Herrera; Jefe de Departamento de la Educación Primaria de la (DME); Subdirector General de Educación; Docente Investigador en la Universidad Nacional de Educación (UNAE); Tengo varios artículos publicados sobre Educación, Didáctica, Pedagogía, TIC y Tecnología Educativa; participé en varios eventos nacionales e internacionales; Participé en varios proyectos de Investigación e innovación educativa; He impartido conferencias y seminarios sobre Tecnología Educativa. 
Arelys García Chávez

e-mail: arelys.garcia@unae.edu.ec

Nacida en Matanzas, Cuba, el 20 de febrero del año

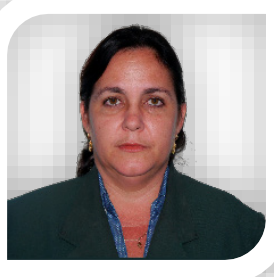

1976. Doctorada en Ciencias Pedagógicas;

Especialista de Postgrado en Trabajo Social

Comunitario en la Mención Dirección; Máster en

Ciencias de la Educación en la Mención Secundaria

Básica y graduada con Título de Oro de la Licenciatura

en Educación en la Especialidad Informática; Es docente investigadora de la Universidad Nacional de Educación (UNAE), en Ecuador; Profesora en la empresa de desarrollo de productos informáticos y audiovisuales (CINESOFT), del Ministerio de Educación (MINED), de la República de Cuba; Es autora de publicaciones vinculadas con la Educación; He dirigido proyectos educativos y tesis en esta área. 


\section{Oscar Antonio Martínez Molina \\ e-mail: oscar.martinez@unae.edu.ec}

Nacido es San Cristóbal, estado Táchira, Venezuela,

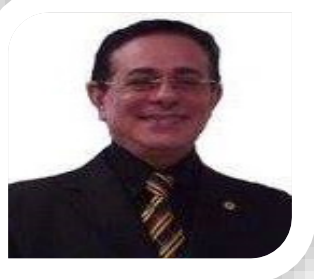

el 12 de octubre del año 1952. Residenciado en Cuenca, Ecuador. Licenciado en Educación Mención Orientación Educativa y Profesional en la Universidad de Los Andes (ULA), Extensión Táchira; Magíster en Ciencias de la Educación Superior, Mención Andragogía en la Universidad Nacional Experimental de Los Llanos Occidentales Ezequiel Zamora (UNELLEZ); Doctor en Educación Mención Summa Cum Laude en la Universidad de Málaga (UMA), España; Postdoctor en Estudios Libres de la Universidad Fermín Toro (UFT); Diplomado en Educación Abierta y a distancia en la Universidad Fermín Toro (UFT); Maestría Experto avanzado en E-learning en la Fundación para la Actualización Tecnológica de Latinoamérica (FATLA); Maestría Experto en Tecnología Educativa nivel avanzado en la Fundación para la Actualización Tecnológica de Latinoamérica (FATLA); Profesor Jubilado de la Universidad Nacional Abierta (UNA); Categoría Académica de Titular; Director de tesis doctorales y de maestría; Representante del convenio Editorial Académica EspañolaCOBAIND; Coordinador de la línea de investigación en Educación en la Universidad Nacional Experimental Politécnica de la Fuerza Armada Bolivariana (UNEFA), Barinas; Presidente y Fundador del COBAID; Profesor investigador del Programa de Estímulo a la Innovación e Investigación en categoría "A-2"; Docente investigación de la Universidad Nacional de Ecuador (UNAE), de Ecuador.

El contenido de este manuscrito se difunde bajo una Licencia de Creative Commons ReconocimientoNoComercial-Compartirlgual 4.0 Internacional 